\title{
A 13-year-old Girl with Hypercapnic Respiratory Failure
}

\author{
AJ Mahendran ${ }^{1}$, Nitesh Gupta ${ }^{2}$, Pranav Ish ${ }^{3}$, Shibdas Chakrabarti $^{4}$
}

\begin{abstract}
Late-onset congenital central hypoventilation syndrome (LO-CCHS) is a rare entity, with scant literature. In view of low prevalence and poor awareness in both patients and doctors, it is often diagnosed late or, worse, misdiagnosed as an obstructive airway disease. Timely diagnosis of central hypoventilation is necessary to prevent hypercapnia, acidosis, and hypoxemia, which can adversely affect the cardiovascular and neurocognitive function. Here we report an interesting case of a young nonobese female who had history of multiple intensive care unit (ICU) admissions for her hypercapnic respiratory failure.

Keywords: Hypoventilation, Idiopathic, Noninvasive ventilation.

Indian Journal of Sleep Medicine (2019): 10.5005/jp-journals-10069-0044
\end{abstract}

\section{Case Description}

A 13-year-old female, born of nonconsanguineous marriage presented with early morning headache, easy fatigability, and dyspnea on exertion. She had been admitted four times in past 2 years in other hospitals with hypercapnic respiratory failure requiring noninvasive ventilation (NIV). There was no history of chronic cough, sputum, wheeze, or chest pain. There was no history suggestive of recurrent childhood pneumonia, delayed milestones, or poor performance at school. Her family did not report her to have any snoring or witnessed apnea during sleep. Clinical examination revealed a sleepy child with height of $136 \mathrm{~cm}$, weight of $38 \mathrm{~kg}$, and a body mass index (BMI) of $20.5 \mathrm{~kg} / \mathrm{m}^{2}$. Her vital signs revealed a pulse rate of 68 beats/minute; respiratory rate of $12 /$ minute, and body temperature of $36^{\circ} \mathrm{C}$. The arterial blood gas analysis showed $\mathrm{pO}_{2}-61 \mathrm{~mm} \mathrm{Hg}, \mathrm{pCO}_{2}-50 \mathrm{~mm} \mathrm{Hg}, \mathrm{HCO}_{3}-29 \mathrm{mmol} / \mathrm{L}$, and $\mathrm{pH}$ 7.34 on room air. The complete blood count, organ functions, chest $X$-ray, thyroid function tests, and serum cortisol levels were also within the normal range. A spirometry, CT chest, CT pulmonary angiography, and maximal inspiratory and expiratory pressure (MIP/ MEP) were normal. In further evaluation, her echocardiography, MRI brain with spinal cord, nerve conduction studies, and laryngoscopy were normal.

Polysomnography (PSG) level 1 under transcutaneous $\mathrm{CO}_{2}$ monitoring $\left(\mathrm{tcpCO} \mathrm{CO}_{2}\right.$ ) was performed (Fig. 1)

The patient was diagnosed as late-onset congenital central hypoventilation syndrome (LO-CCHS).

The patient had hypercapnic respiratory failure in the arterial blood gas analysis, but with a preserved alveolar-arterial gradient suggestive of pure hypoventilation. Hypoventilation is defined as a fall in ventilation leading to rise in $\mathrm{pCO}_{2}$ by $10 \mathrm{~mm} \mathrm{Hg}$ in a polysomnography or to a value more than $45 \mathrm{~mm} \mathrm{Hg},{ }^{1}$ which was documented in our patient using transcutaneous $\mathrm{pCO}_{2}(\mathrm{tcpCO})$ rising from 45 to $65 \mathrm{~mm} \mathrm{Hg}$ in the PSG. There was no obstructive apnea, hypopnea, or abdominal paradox. There were frequent arousal and prolonged desaturations during sleep with lowest saturation recorded being $65 \%$. The patient had history of multiple past admissions for her hypercapnic respiratory failure but had not undergone a PSG. In absence of any identifiable pulmonary, cardiac, neuromuscular disease, brainstem disorder, chest wall disorder, chronic obstructive pulmonary disease, chronic drug abuse, or obesity hypoventilation, this patient was diagnosed with LO-CCHS.
${ }^{1-4}$ Department of Pulmonary, Critical Care and Sleep Medicine, Vardhman Mahavir Medical College and Safdarjung Hospital, New Delhi, India

Corresponding Author: Pranav Ish, Department of Pulmonary, Critical Care and Sleep Medicine, Vardhman Mahavir Medical College and Safdarjung Hospital, New Delhi, India, Phone: +91 9958356000, e-mail: pranavish2512@gmail.com

How to cite this article: Mahendran AJ, Gupta N, Ish $P$, et al. A 13-year-old Girl with Hypercapnic Respiratory Failure. Indian J Sleep Med 2019;14(4):76-78.

Source of support: Nil

Conflict of interest: None

The genetic mutation analysis could not be carried out in view of nonavailability in our hospital and nonaffordability of the patient for the same for paid labs. There was no family history of similar such complaints in siblings and parents of the patient.

\section{Discussion}

Late-onset congenital central hypoventilation syndrome is characterized by adequate ventilation while the patient is awake but hypoventilation with preserved respiratory rates and shallow breathing (decreased tidal volume) during sleep. ${ }^{2}$ While asleep, such patients develop progressive hypercapnia and hypoxemia with variable ventilatory sensitivity to both. Gradually, there is progression from nocturnal to diurnal hypoventilation. ${ }^{3}$

Children with CCHS usually present in infancy with appearance of cyanosis during sleep with no tachypnea or awakening. ${ }^{4}$ Such children require tracheostomy and ventilatory support. Elder children having central alveolar hypoventilation, who can cooperate, can be managed with noninvasive ventilation. Being a lifelong disease, management requires a diligent effort and support on the part of the parents, family members, home health-care personnel, and physicians. Our patient was titrated with NIV using tcpCO $\mathrm{C}_{2}$ as the guide and corrected sleep-induced hypoventilation. With regular use NIV, a daytime $\mathrm{pCO}_{2}$ of $41 \mathrm{~mm} \mathrm{Hg}$ was achieved after 4 weeks. The patient's early-morning headaches and fatigue reduced significantly with no further hospital admission. Her ABG on follow-up showed a $\mathrm{pH}$ of 7.412, $\mathrm{pCO}_{2} 41 \mathrm{~mm} \mathrm{Hg}$, and $\mathrm{pO}_{2} 79 \mathrm{~mm} \mathrm{Hg}$ 


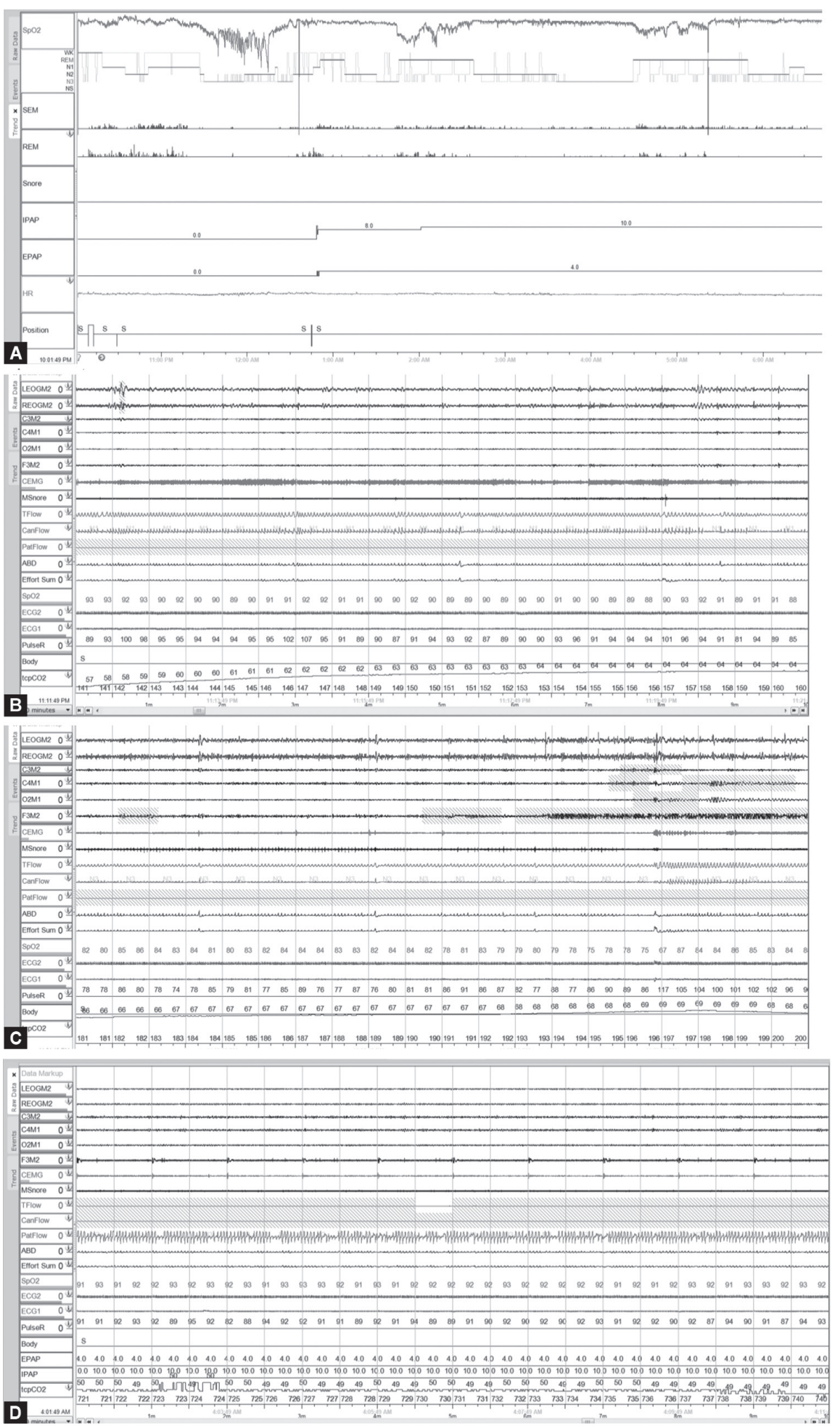

Figs 1 A to D: (A) Hypnogram showing persistent desaturation suggestive of hypoventilation; (B and C) Polysomnography 10-minute window with epoch 141-160 showing no obstructive event and persistent rise in transcutaneous $\mathrm{CO}_{2}$ from 57 to $64 \mathrm{~mm} \mathrm{Hg}$, which further rises in epoch $181-200$ to $69 \mathrm{~mm} \mathrm{Hg}$ confirming hypoventilation; (D) Polysomnography 10-minute window with epoch 721-740 showing correction in transcutaneous $\mathrm{CO}_{2}$ to 49 using bilevel positive airway pressure with inspiratory pressure of $10 \mathrm{~mm} \mathrm{Hg}$ and expiratory pressure of $4 \mathrm{~mm} \mathrm{Hg}$. Only correction of nocturnal hypoventilation to an awake $\mathrm{CO}_{2}$ value was targeted. $\mathrm{SpO}_{2}$, saturation of oxygen in percent; SEM, slow eye movement; REM, rapid eye movement; IPAP, inspiratory positive airway pressure; EPAP, expiratory positive airway pressure; HR, heart rate; position S, supine; REOGM2 and LEOGM2, right and left electrooculograph; F3M2, C4M1, C3M2, and O2M1, electroencephalograph; CEMG, chin electromyography; MSnore, snoring; T flow, thermistor flow; Can flow, nasal cannula flow; PatFlow, patient flow using a positive airway pressure device; THO, thoracic muscle effort; $\mathrm{ABD}$, abdominal muscle effort; Effort sum, thorax and abdominal effort sum; PulseR, pulse rate; $\mathrm{SpO}_{2}$, oxygen saturation; Body, body position; tcp $\mathrm{CO}_{2}$, transcutaneous measurement of $\mathrm{CO}_{2}$; ECG 1,2, electrocardiograph 
Our case highlights the importance of timely diagnosis and proper management with NIV for patients with LO-CCHS as this can help to reduce the associated morbidity. ${ }^{5}$

\section{Declaration of Patient Consent}

The authors certify that they have obtained all appropriate patient consent forms. In the form, the patient(s) has/have given his/her/ their consent for his/her/their images and other clinical information to be reported in the journal.

The patient understands that his name and initials will not be published and due efforts will be made to conceal his identity, but anonymity cannot be guaranteed.

\section{References}

1. Brown LK. Hypoventilation syndromes. Clin Chest Med 2010;31(2): 249-270. DOI: 10.1016/j.ccm.2010.03.002.

2. Dosier LBM, Vaughn BV, Fan Z. Sleep disorders in childhood neurogenetic disorders. Children (Basel) 2017;4(9):82.

3. Magalhães J, Madureira N, Medeiros R, et al. Late-onset congenital central hypoventilation syndrome and a rare $\mathrm{PHOX} 2 \mathrm{~B}$ gene mutation. Sleep Breath 2015;19(1):55-60. DOI: 10.1007/s11325-014-0996-7.

4. Gothi $D$, Joshi JM. Late onset hypoventilation syndrome: is there a spectrum of idiopathic hypoventilation syndromes? Indian J Chest Dis Allied Sci 2005;47(4):293-298.

5. Marcus CL. Sleep-disordered breathing in children. Am J Respir Crit Care Med 2001;164(1):16-30. DOI: 10.1164/ajrccm.164.1.2008171. 